\title{
Biliary Tract Infection or Colonization with Elizabethkingia meningoseptica after Endoscopic Procedures Involving the Biliary Tract
}

\author{
Zhiyong Zong
}

\begin{abstract}
Objective This study was performed to investigate the clinical significance of detecting Elizabethkingia meningoseptica in the bile.

Methods We herein report a case series of biliary colonization or infection with E. meningoseptica. Twenty patients with E. meningoseptica recovered from the bile were treated at a 4,300-bed teaching hospital in China between January 2009 and December 2012. The clinical information for the cases of E. meningoseptica recovered from the bile and the microbiological data of the E. meningoseptica isolates were examined.

Results Most of the 20 patients were not immunocompromised, although they had cholelithiasis and had recently received antimicrobial agents. All cases were treated with indwelling nasobiliary tubes and endoscopic retrograde cholangiopancreatography and/or endoscopic sphincterotomy prior to the detection of $E$. meningoseptica in the bile. The average time between the placement of the nasobiliary tube and the detection of E. meningoseptica in the bile was 6.6 days. E. meningoseptica caused cholangitis in five cases, one of which also involved secondary septicemia, and colonized the nasobiliary tubes or biliary tract in the remaining 15 cases. All but two patients recovered and were discharged. Two patients died of septicemia; E. meningoseptica and Escherichia coli were the causative pathogens in one case and other organisms were the causative pathogens in the other.

Conclusion E. meningoseptica is an unusual causative pathogen of healthcare-associated cholangitis. Cholangitis resulting from this bacterium is generally associated with good outcomes, although secondary septicemia can be life-threatening.
\end{abstract}

Key words: Elizabethkingia meningoseptica, Chryseobacterium meningosepticum, cholangitis, biliary tract infection, colonization

(Intern Med 54: 11-15, 2015)

(DOI: 10.2169/internalmedicine.54.3034)

\section{Introduction}

Elizabethkingia meningoseptica, named Chryseobacterium meningosepticum prior to 2005 (1) and Flavobacterium meningosepticum prior to 1994 (2), is a saprophytic Gramnegative bacterium that was widely distributed in the environment. As an opportunistic pathogen, E. meningoseptica has been reported to cause a variety of infections, including abdominal infections, endocarditis, eye infections, meningitis, pneumonia, osteomyelitis, septic arthritis, septicemia and skin and soft tissue infections (3-6). Infections caused by $E$. meningoseptica are primarily healthcare associated and occur in newborns and immunocompromised adults (3). A few outbreaks due to this opportunistic pathogen have also been reported (7-10). Furthermore, recovery of E. meningoseptica from bile samples has been documented $(3,11)$, although it remains rare. Furthermore, the clinical significance of identifying E. meningoseptica in the bile is unclear. A total of 20 patients with E. meningoseptica recovered from bile samples were identified at West China Hospital. We herein report the characteristics of these patients. 


\section{Materials and Methods}

\section{Patients and setting}

West China Hospital of Sichuan University is a 4,300-bed referral teaching hospital in Chengdu, Sichuan province, western China. Between January 2009 and December 2012, there was a total of 20 patients in whom E. meningoseptica was recovered from bile samples. A case investigation form was used to collect the demographic data, medical history, underlying diseases, clinical manifestations, laboratory results, treatments and outcomes of the cases.

\section{Bacterial isolates and antimicrobial susceptibility testing}

The bile samples were generally collected by puncturing nasobiliary tubes using needles and syringes and processed using standard laboratory protocols. Species identification and in vitro susceptibility testing of E. meningoseptica isolates were performed using the Vitek II (bioMérieux, Durham, USA) automated system. The interpretation of the minimum inhibitory concentrations was made based on standards for non-Enterobacteriaceae set by the Clinical and Laboratory Standards Institute (12).

\section{Definitions}

The clinical significance, i.e., infection or colonization, of E. meningoseptica isolates was determined based on the presence of a biliary tract infection according to clinical manifestations, laboratory data and ultrasound, computerized tomography or magnetic resonance imaging findings obtained within two days of the collection of the specimens from which E. meningoseptica was isolated. Cholangitis and cholecystitis were diagnosed using the criteria of the Tokyo guidelines $(13,14)$. Healthcare-associated infection or colonization was defined as the acquisition of E. meningoseptica after 48 hours of admission to our hospital. Antimicrobial therapy for E. meningoseptica was considered to be appropriate if the isolates were susceptible to the agents administered. Death due to E. meningoseptica infection was defined as death within two weeks of infection in the absence of other known causes (15).

\section{Results}

The 20 patients included 12 men and eight women, 22 to 89 years of age, with a median age of 52 years (Table 1). Only four patients were older than 65 years. Fifteen of the 20 patients were not in an obvious immunocompromised condition, while the remaining five patients had diabetes ( $\mathrm{n}=$ $2)$ or carcinoma $(n=2)$ or had undergone liver transplantation $(\mathrm{n}=1)$. Most $(17 / 20)$ of the patients had biliary tract illnesses, while half had pancreatitis. Among the biliary tract illnesses, cholelithiasis was the most common condition, observed in 13 cases, followed by carcinoma, anomalous pan- creaticobiliary ductal union and common bile duct stricture, present in one or two cases each. One patient had biliary tract obstruction; however, the exact cause remained unclear. Fourteen of the 20 patients received antimicrobial agents, mostly cephalosporins (nine cases) or carbapenems (four cases), within one month before collection of the specimen that grew E. meningoseptica. All of the patients were treated with indwelling endoscopic nasobiliary drainage (ENBD) tubes and endoscopic retrograde cholangiopancreatography (ERCP) or endoscopic sphincterotomy (EST) or both prior to the detection of E. meningoseptica in the bile. Some patients also underwent other operations involving the biliary tract, such as choledochotomy, choledochoscopy and cholecystectomy (Table 1). The time between the placement of the nasobiliary tube and the detection of E. meningoseptica in the bile ranged from 0 (the same day) to 24 days, at 6.6 days on average.

In all but two cases, E. meningoseptica was the sole microorganism recovered from a single bile specimen; the remaining two patients had either Acinetobacter baumannii or Stenotrophomonas maltophilia isolated together with $E$. meningoseptica from the same bile specimen. Three patients had E. meningoseptica recovered from two bile specimens collected at different time points, and E. meningoseptica was isolated from blood in one (case no. 13) of these three cases. Four patients had other bacteria, including A. baumannii in all four cases, Enterococcus faecium in three cases and Klebsiella pneumoniae in two cases, recovered from bile specimens collected at different time points. Two of these four patients also had blood cultures that grew other bacteria, either Escherichia coli or E. faecium. All E. meningoseptica isolates were considered to be healthcare associated.

E. meningoseptica was consider to be causative pathogen of cholangitis in five cases and as a microorganism colonized in the nasobiliary tube or biliary tract in the remaining 15 cases. All five patients with cholangitis due to E. meningoseptica met the criteria of the Tokyo guidelines for a definitive diagnosis of cholangitis, including systemic inflammation, cholestasis and imaging of biliary dilatation, stones or stricture (13). Among the five cases of cholangitis, $E$. meningoseptica was either the only microorganism identified or the first bacterium recovered and was isolated from specimens collected within two days of the onset of cholangitis. One patient (case no. 13) developed septicemia caused by $E$. meningoseptica secondary to cholangitis. In 12 of the 20 cases, E. meningoseptica was considered to have colonized the nasobiliary tube or biliary tract, rather than caused an infection, due to the absence of biliary tract infection. Two patients were admitted to our hospital due to cholangitis, and their manifestations of the disease were gradually resolving at the time at which the specimens that grew E. meningoseptica were collected. Therefore, E. meningoseptica was regarded to have colonized the patients in both cases. One patient (case no. 15) developed cholangitis beyond 48 hours after admission and was therefore considered to have a 
Table 1. Characteristics of the Patients with E. Meningoseptica (Em) Recovered from the Bile

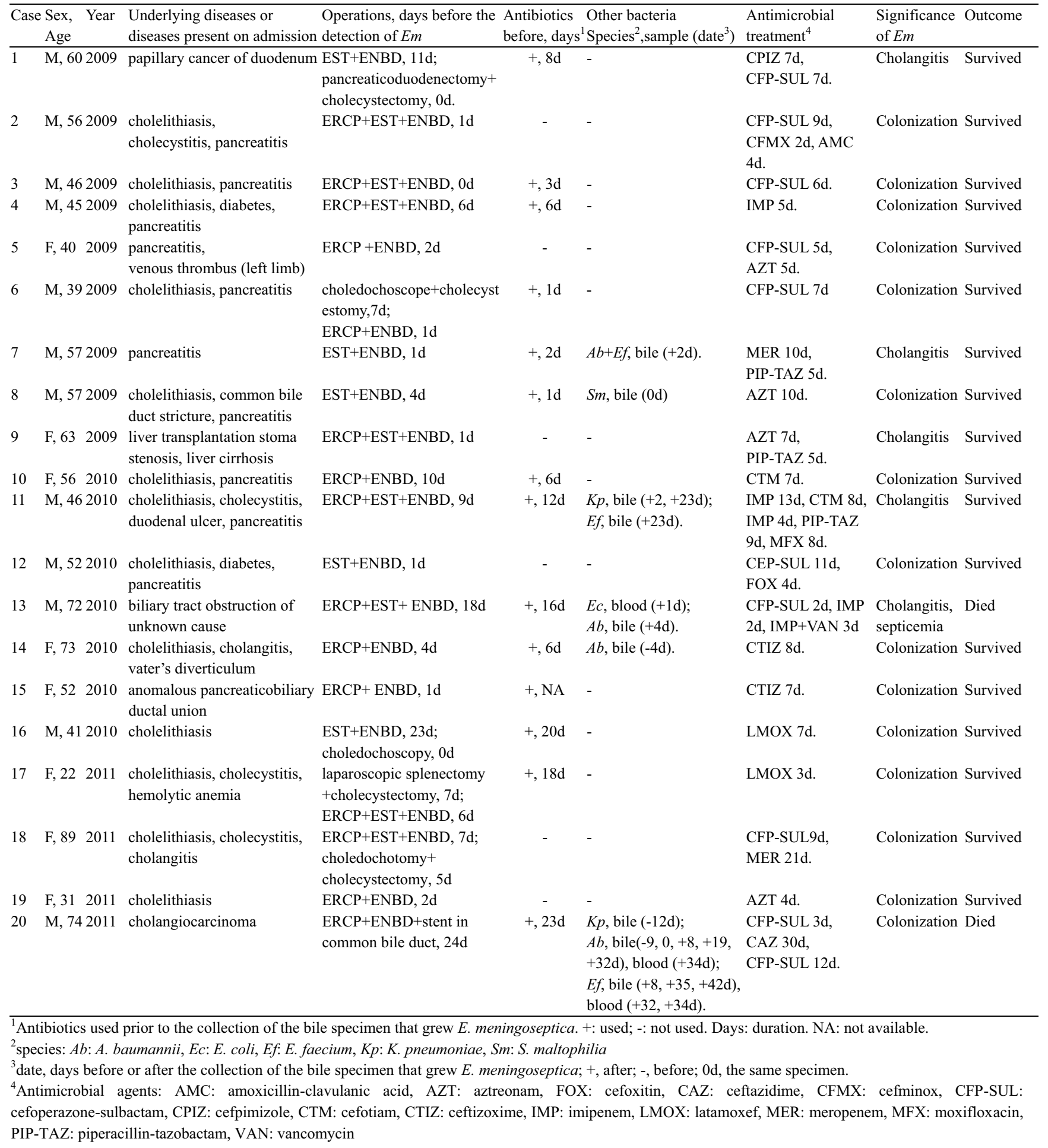

healthcare-associated infection. This patient also had $K$. pneumoniae and A. baumannii isolated from bile specimens prior to the recovery of E. meningoseptica, and his condition had not deteriorated within two days before or after the collection of the specimen that grew E. meningoseptica. In addition, A. baumannii and E. faecium were repeatedly identified in multiple bile specimens collected at different time points, and the two bacteria were recovered from blood cultures. Therefore, E. meningoseptica was not considered to be the causative pathogen of the healthcare-associated cho- langitis observed in case no. 15.

Although E. meningoseptica had simply colonized the patients in most cases, the affected patients usually had pancreatitis or cholecystitis, and all patients with E. meningoseptica colonization or infection were administered antimicrobial agents. However, none of the agents were appropriate with respect to the susceptibility results. Nonetheless, all but two patients recovered and were discharged. Two patients died of septicemia; E. meningoseptica and Escherichia coli were the causative pathogens in case no. 15, and $A$. 
Table 2. Characteristics of the Septicemia Patients with E. Meningoseptica Secondary to Biliary Tract Infection

\begin{tabular}{|c|c|c|c|c|}
\hline Sex Age Underlying diseases & Biliary tract infection & Antimicrobial & Outco & Reference \\
\hline M 56 liver transplantation & cholangitis & not available & Survi & (3) \\
\hline $\begin{array}{c}\text { F } 66 \text { diabetes, coronary artery } \\
\text { disease }\end{array}$ & no information & $\mathrm{CIP}^{1}$ & Survi & (16) \\
\hline F 90 congestive heart failure & no information & CIP & Died & (16) \\
\hline M 72 biliary tract obstruction & cholangitis & $\begin{array}{l}\text { CFP-SUL, } \\
\text { IMP, VAN }\end{array}$ & Died & This study \\
\hline
\end{tabular}

${ }^{1}$ CIP: ciprofloxacin.

baumannii and E. faecium were the causative pathogens in case no. 20. Therefore, only one of the 20 patients died of infection due to E. meningoseptica.

All isolates in this study were resistant to aminoglycosides (amikacin, gentamicin and tobramycin), penicillins (piperacillin and ticarcillin-clavulanic), cephalosporins (cefotaxime, ceftriaxone, ceftazidime and cefepime), aztreonam, imipenem and fluoroquinolones (ciprofloxacin and levofloxacin). All but one were also resistant to piperacillintazobactam, with the remaining isolate being intermediate. Isolates from 11 cases were susceptible to trimethoprimsulfamethoxazole, while the remaining isolates were resistant to these drugs.

\section{Discussion}

Recovering E. meningoseptica from the bile is unusual. For example, this bacterium accounted for $2.6 \%$ of microorganisms isolated from bile cultures obtained between January 2009 and December 2012 at West China Hospital, and only a few cases of E. meningoseptica recovered from the bile have been reported in the literature $(3,16)$. Nonetheless, E. meningoseptica can cause biliary tract infections and septicemia secondary to cholangitis, although, in most cases, this bacterium results in colonization rather than infection. It has been documented that E. meningoseptica is able to colonize several body sites and medical tubes, including the oropharynx (17), respiratory secretions (18), rectum (17), aerosol tubes (17) and endotracheal tubes (9, 17); however, colonization of nasobiliary tubes and/or the biliary tract has not been previously described. Three cases $(3,16)$ of E. meningoseptica septicemia secondary to biliary tract infection have been reported in the literature (Table 2). One of these patients had cholangitis, as in case no. 13 in the present study (3), whereas the type of biliary tract infection was not specified and no information regarding whether E. meningoseptica was isolated from the bile was provided in the other two cases (16). Two of the three patients survived after receiving antimicrobial therapy, while the remaining patient died despite receiving treatment with an agent to which the E. meningoseptica isolate was susceptible. The operations applied in the present study involved the biliary tract, in particular the use of an indwelling nasobiliary tube appeared to be the most important risk factor for E. meningoseptica colonization or infection, as all patients in this study received an indwelling nasobiliary tube and ERCP or EST, while some patients also received choledochoscopy or cholecystectomy. Of note, unlike previous reports of E. meningoseptica infection or colonization at other sites, most of the present patients with biliary tract colonization or infection were not neonates, immunocompromised adults or elderly.

E. meningoseptica is intrinsically resistant to most commonly used antimicrobial agents; therefore, options for antimicrobial therapy against this opportunistic pathogen are limited. In previous reports $(6,9,19-21)$, the clinical isolates of E. meningoseptica were always resistant to aminoglycosides and almost all $\beta$-lactams, except for piperacillintazobactam, and usually susceptible to trimethoprimsulfamethoxazole and fluoroquinolones. However, unlike that found in most previous reports $(6,19)$, all isolates in this study were also resistant to fluoroquinolones (ciprofloxacin and levofloxacin), and none of the isolates were susceptible to piperacillin-tazobactam. In addition, almost half of the isolates were resistant to trimethoprimsulfamethoxazole, making the selection of agents for appropriate antimicrobial treatment particularly difficult. Nonetheless, four of the five patients with E. meningoseptica infection survived, suggesting that host factors are critical determinants of the outcome in cases of E. meningoseptica infection, as previously suggested (16), and that treatments other than antimicrobial agents, such as drainage, are also critical. In addition, the susceptibility breakpoints for unusual opportunistic non-fermenting Gram-negative pathogens, such as $E$. meningoseptica, should be verified.

The present study is a retrospective analysis and therefore is associated with several limitations. For instance, the isolates were not stored for further study of their clonal relatedness, strain type and susceptibility to several antimicrobial agents that may be effective, such as vancomycin, linezolid, rifampicin and minocycline. Furthermore, no follow-up bile specimens were collected in most cases; therefore, the duration of colonization of E. meningoseptica could not be determined. E. meningoseptica can cause outbreaks as an opportunistic pathogen of healthcare-associated infections (22-24), and it is possible that the isolates reported in this series are closely related and have caused outbreaks. However, due to the absence of data for strain typing, it is impossible to confirm or exclude the presence of any outbreaks. 
In summary, E. meningoseptica is an unusual pathogen causing colonization as well as healthcare-associated cholangitis after operations involving the biliary tract and indwelling nasobiliary tubes. Biliary colonization or infection with E. meningoseptica are primarily observed in patients who are neither elderly nor immunocompromised. Although the E. meningoseptica isolated from the bile in the present study was found to be resistant to most commonly used antimicrobial agents, in vitro susceptibility findings are not usually relevant to the clinical outcome, and all cases of cholangitis caused by this bacterium without secondary septicemia resolved in the present study. Although E. meningoseptica septicemia can develop secondary to cholangitis, it remains a very rare and life-threatening infection.

The author states that he has no Conflict of Interest (COI).

\section{References}

1. Kim KK, Kim MK, Lim JH, Park HY, Lee ST. Transfer of Chryseobacterium meningosepticum and Chryseobacterium miricola to Elizabethkingia gen. nov. as Elizabethkingia meningoseptica comb. nov. and Elizabethkingia miricola comb. nov. Int J Syst Evol Microbiol 55: 1287-1293, 2005.

2. Vandamme P, Bernardet JF, Segers P, Kersters K, Holmes B. New perspectives in the classification of the flavobacteria: description of Chryseobacterium gen. nov., Bergeyella gen. nov., and Empedobacter nom. rev. Int J Syst Bacteriol 44: 827-831, 1994.

3. Bloch KC, Nadarajah R, Jacobs R. Chryseobacterium meningosepticum: an emerging pathogen among immunocompromised adults. Report of 6 cases and literature review. Medicine (Baltimore) 76: 30-41, 1997.

4. Lee $\mathrm{CH}$, Lin WC, Chia JH, et al. Community-acquired osteomyelitis caused by Chryseobacterium meningosepticum: case report and literature review. Diagn Microbiol Infect Dis 60: 89-93, 2008.

5. Kumar R, Stephens JL. Septic arthritis caused by Chryseobacterium meningosepticum in an elbow joint prosthesis. South Med J 97: 74-76, 2004.

6. Hsu MS, Liao CH, Huang YT, et al. Clinical features, antimicrobial susceptibilities, and outcomes of Elizabethkingia meningoseptica (Chryseobacterium meningosepticum) bacteremia at a medical center in Taiwan, 1999-2006. Eur J Clin Microbiol Infect Dis 30: 1271-1278, 2011.

7. Tekerekoglu MS, Durmaz R, Ayan M, Cizmeci Z, Akinci A. Analysis of an outbreak due to Chryseobacterium meningosepticum in a neonatal intensive care unit. New Microbiol 26: 57-63, 2003.

8. Ceyhan M, Yildirim I, Tekeli A, et al. A Chryseobacterium meningosepticum outbreak observed in 3 clusters involving both neonatal and non-neonatal pediatric patients. Am J Infect Control 36: 453-457, 2008.
9. Maraki S, Scoulica E, Manoura A, Papageorgiou N, Giannakopoulou C, Galanakis E. A Chryseobacterium meningosepticum colonization outbreak in a neonatal intensive care unit. Eur J Clin Microbiol Infect Dis 28: 1415-1419, 2009.

10. Brown RB, Phillips D, Barker MJ, Pieczarka R, Sands M, Teres D. Outbreak of nosocomial Flavobacterium meningosepticum respiratory infections associated with use of aerosolized polymyxin B. Am J Infect Control 17: 121-125, 1989.

11. Igari J, Kosakai N, Oguri T. Clinical and bacteriological study on Flavobacterium meningosepticum. Jpn J Antibiot 30: 242-249, 1977.

12. Clinical and Laboratory Standards Institute. Performance Standards for Antimicrobial Susceptibility Testing; Eighteen Informational Supplement: M100-S18, 2008.

13. Kiriyama S, Takada T, Strasberg SM, et al. New diagnostic criteria and severity assessment of acute cholangitis in revised Tokyo Guidelines. J Hepatobiliary Pancreat Sci 19: 548-556, 2012.

14. Yokoe M, Takada T, Strasberg SM, et al. New diagnostic criteria and severity assessment of acute cholecystitis in revised Tokyo Guidelines. J Hepatobiliary Pancreat Sci 19: 578-585, 2012.

15. Liao CH, Sheng WH, Chen YC, Hung CC, Wang JT, Chang SC. Predictive value of the serum bactericidal test for mortality in patients infected with multidrug-resistant Acinetobacter baumannii. J Infect 55: 149-157, 2007.

16. Hung PP, Lin YH, Lin CF, Liu MF, Shi ZY. Chryseobacterium meningosepticum infection: antibiotic susceptibility and risk factors for mortality. J Microbiol Immunol Infect 41: 137-144, 2008.

17. Hazuka BT, Dajani AS, Talbot K, Keen BM. Two outbreaks of Flavobacterium meningosepticum type $\mathrm{E}$ in a neonatal intensive care unit. J Clin Microbiol 6: 450-455, 1977.

18. Hoque SN, Graham J, Kaufmann ME, Tabaqchali S. Chryseobacterium (Flavobacterium) meningosepticum outbreak associated with colonization of water taps in a neonatal intensive care unit. J Hosp Infect 47: 188-192, 2001.

19. Lin PY, Chu C, Su LH, Huang CT, Chang WY, Chiu CH. Clinical and microbiological analysis of bloodstream infections caused by Chryseobacterium meningosepticum in nonneonatal patients. $\mathrm{J}$ Clin Microbiol 42: 3353-3355, 2004.

20. Ceyhan M, Celik M. Elizabethkingia meningosepticum (Chryseobacterium meningosepticum) Infections in Children. Int $\mathrm{J}$ Pediatr 2011: 215237, 2011.

21. Chiu $\mathrm{CH}$, Waddingdon $\mathrm{M}$, Greenberg $\mathrm{D}$, Schreckenberger PC, Carnahan AM. Atypical Chryseobacterium meningosepticum and meningitis and sepsis in newborns and the immunocompromised, Taiwan. Emerg Infect Dis 6: 481-486, 2000.

22. Jean SS, Lee WS, Chen FL, Ou TY, Hsueh PR. Elizabethkingia meningoseptica: an important emerging pathogen causing healthcare-associated infections. J Hosp Infect 86: 244-249, 2014.

23. Weaver KN, Jones RC, Albright R, et al. Acute emergence of Elizabethkingia meningoseptica infection among mechanically ventilated patients in a long-term acute care facility. Infect Control Hosp Epidemiol 31: 54-58, 2010.

24. Issack MI, Neetoo Y. An outbreak of Elizabethkingia meningoseptica neonatal meningitis in Mauritius. J Infect Dev Ctries 5: 834839, 2011.

(C) 2015 The Japanese Society of Internal Medicine

http://www.naika.or.jp/imonline/index.html 